\title{
QUIESCENCE AS AN EXPLANATION OF GOMPERTZIAN TUMOR GROWTH REVISITED
}

\author{
E. O. Alzahrani ${ }^{1}$, Asim Asiri ${ }^{1}$, M. M. El-Dessoky ${ }^{1,2}$, And Y. Kuang ${ }^{3}$ \\ ${ }^{1}$ Department of Mathematics, Faculty of Science \\ King Abdulaziz University, P. O. Box 80203, Jeddah 21589, Saudi Arabia \\ ${ }^{2}$ Department of Mathematics, Faculty of Science \\ Mansoura University, Mansoura 35516, Egypt \\ ${ }^{3}$ School of Mathematical and Statistical Sciences \\ Arizona State University, Tempe, AZ 85287, USA
}

\begin{abstract}
Gompertz's empirical equation remains the most popular one in describing cancer cell population growth in a wide spectrum of biomedical situations due to its good fit to data and simplicity. Many efforts were documented in the literature aimed at understanding the mechanisms that may support Gompertz's elegant model equation. One of the most convincing efforts was carried out by Gyllenberg and Webb. They divide the cancer cell population into the proliferative cells and the quiescent cells. In their two dimensional model, the dead cells are assumed to be removed from the tumor instantly. In this paper, we modify their model by keeping track of the dead cells remaining in the tumor. We perform mathematical and computational studies on this three dimensional model and compare the model dynamics to that of the model of Gyllenberg and Webb. Our mathematical findings suggest that if an avascular tumor grows according to our three-compartment model, then as the death rate of quiescent cells decreases to zero, the percentage of proliferative cells also approaches to zero. Moreover, a slow dying quiescent population will increase the size of the tumor. On the other hand, while the tumor size does not depend on the dead cell removal rate, its early and intermediate growth stages are very sensitive to it.
\end{abstract}

\section{INTRODUCTION}

Tumor growth models have their historical roots in the work of Gompertz (1825). The Gompertz model was first employed in the paper of Laird (1964) to model real tumor growth. Ever since, Gompertz's empirical equation remains the most popular one in describing cancer cell population growth in a wide

1991 Mathematics Subject Classification. Primary: 34K20, 92C50; Secondary: 92D25.

Key words and phrases. Tumor model, quiescence, proliferation, steady state, stability.

Corresponding author: Yang Kuang, kuang@asu.edu, phone 480-965-6915, fax 480-9658119 . 
spectrum of bio-medical situations due to its good fit to data and simplicity (Norton (1976)). Many efforts were documented in the literature aimed at understanding the mechanisms that may support Gompertz's elegant model equation (Frenzen and Murray (1986), Gyllenberg and Webb (1989), Marusic and Vuk-Pavlovic (1993), Marusic et al. (1994), Kozusko and Bajzer (2003), Thalhauser et al. (2009)). The key aspect of the approach of these existing efforts is to divide the cancer cell population into the proliferating cells and the quiescent cells, or the proliferating cell and dispersing cells.

In the beginning, a tumor often grows in approximately a spherical form. If the tumor fails to produce enough signaling proteins such as vascular endothelial growth factor (VEGF) for angiogenesis, then the tumor can only grow to a certain size with available nutrient supplies. Indeed, most tumors exhibit a sigmoid growth curve in the early stage. For this reason, many modelers simply employ the well-known logistic equation

$$
d N / d t=r N(1-N / K)=r N-r N^{2} / K
$$

as an initial model for tumor growth. Here $N$ is the size of the tumor, usually measured as a number of cells or as a volume. $r$ is the growth rate while $r N / K$ can be interpreted as the density dependent death rate. The tumor size is an increasing function that tends to the carrying capacity $K$. Generalizing the logistic model, von Bertalanffy (1957) introduced the equation

$$
d N / d t=f(N)=\alpha N^{\lambda}-\beta N^{\mu}, \quad \lambda<\mu .
$$

to represent tumor growth. This is often referred as the (generalized) von Bertalanffy tumor model. The tumor size is an increasing function that tends to the carrying capacity $(\alpha / \beta)^{1 /(\mu-\lambda)}$. Tumors tend to approach a steady state size in the nutrient-limited growth phase when nutrient is supplied only by diffusion. A particular case of the von Bertalanffy equation is the surface rule model (Bertalanffy (1941)), which states that growth is proportional to surface area $(\lambda=2 / 3)$ since nutrients have to enter through the surface, while death is proportional to the size $(\mu=1)$. In this special case, $\beta$ is the death rate. Notice that, the birth rate of logistic model and the death rate of the von Bertalanffy model (when $\mu=1$ ) are constant.

Gompertz model is arguably also the most important and practical tumor model. Many researchers reported that Gompertz model provided surprisingly good fit to their experimental data on various tumor growths. The key assumption embodied in the Gompertz model is that the cell growth rate decreases exponentially as a function of time.

$$
\frac{d N}{d t}=r(t) N(t), \quad \frac{d r}{d t}=-\operatorname{ar}(t)
$$


Notice that

$$
\frac{d(\ln (N))}{d t}=\frac{1}{N} \frac{d N}{d t}=r(t)=\frac{-1}{a} \frac{d r}{d t}
$$

from which we obtain that for some constant $b$,

$$
\ln (N)=(-r(t)+b) / a
$$

which is equivalent to say that $r(t)=b-a \ln (N)$. This gives us an alternative and more popular form of the Gompertz model

$$
\frac{d N}{d t}=b N-a \ln (N) N=N(b-a \ln (N)) .
$$

Note that this function is not defined for $N=0$, so we must assume that the tumor has a certain size before applying this model. Again, the tumor size is an increasing function that tends to the carrying capacity $K=e^{b / a}$. Using the substitution $u=\ln (N / K)$, we can solve the Gompertz equation with initial condition $N(0)=N_{0}$. We obtain that $N(t)=K e^{A B}$, where $A=\ln \left(N_{0} / K\right)$ and $B=e^{-a t}$. Some researchers have also considered the following so-called generalized Gompertz model (Marusic and Vuk-Pavlovic (1993), Marusic et al. (1994)),

$$
\frac{d N}{d t}=N^{\alpha}(b-a \ln (N))
$$

Marusic and Vuk-Pavlovic (1993) compared the Gompertz model, the generalized Gompertz model, and a host of other one dimensional ODE tumor models (including the above logistic and von Bertalanffy tumor models) to predict growth of multicellular tumor spheroids as paradigms of the prevascular phase of tumor growth. They reported that the Gompertz model is the model with the best prediction power. The generalized Gompertz model is ranked as the second most predictive model. Moreover, the ranking of models was not affected by the applied minimization criteria of weighted least squares, unweighted least squares and fitting to logarithmically transformed data.

While these models have intuitively meaningful parameters, they all ignored the typical three-layer structure manifested by most multicellular tumor spheroids in their later phase (see Fig. 1). Hence, in Marusic et al. (1994), the authors stated that more sophisticated models that incorporate fine tumor structures are needed. This in fact was pursued in a two-compartment model of cancer cells population growth dynamics proposed by Gyllenberg and Webb (1989). In their model, the transition rates between proliferating and quiescent cells are assumed to be non-specified functions of the total population N. As a result, the net inter-compartmental transition rate function is also a function of the total cells. For some special set of parameter values and initial conditions, this net inter-compartmental transition rate function can 


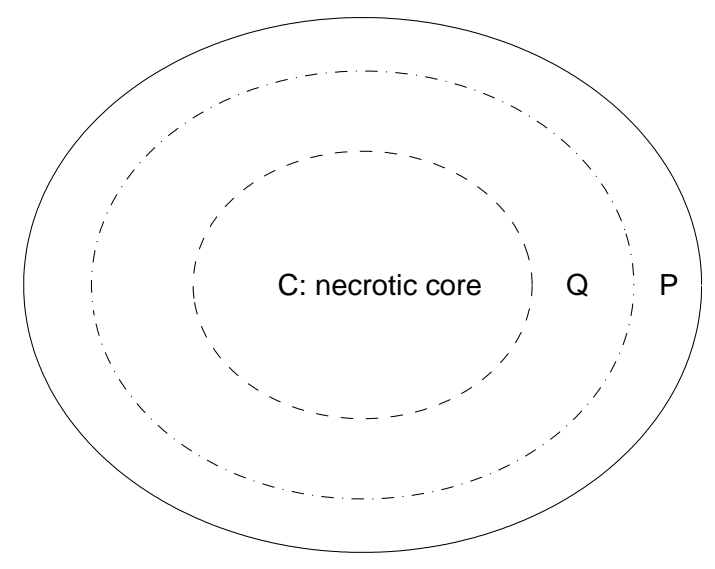

Figure 1. Structure of a typical multicellular tumor spheroid. $\mathrm{Q}=$ quiescent layer, $\mathrm{P}=$ proliferating layer.

be selected to generate the Gompertz growth model. Effectively, this leads to a hybrid model for which explicit analytical solutions for proliferating and quiescent cell populations, and the relations among model parameters can be obtained. The model realistically predicts that the number of proliferating cells may increase along with the total number of cells, but the proliferating fraction appears to be a continuously decreasing function. The net transition rate of cells is shown to retain direction from the proliferating into the quiescent compartment. The death rate parameter for quiescent cell population is shown to be a factor in determining the proliferation level for a particular Gompertz growth curve. However, in this model, the dead cells are assumed to be removed from the tumor instantly which is a drastic simplification of reality and ignores the ubiquitous necrotic core feature of a typical late stage avascular tumor.

In this paper, we modify the model of Gyllenberg and Webb (1989) by keeping track of the dead cells remaining in the tumor. We perform systematical mathematical and computational analysis of the model properties and dynamics. In particular, we compare and contrast our three dimensional model dynamics to that of the two dimensional model of Gyllenberg and Webb (1989).

\section{A THREE-COMPARTMENT MODEL FOR TUMOR GROWTH}

In a typical avascular multicellular tumor spheroid, due to lack of key resources such as nutrient and space, proliferating cells often enter a quiescent 
state where they stop dividing. Quiescent cells may die if the resource limitation persists or return to proliferating state if enough resources can be obtained in a given period. Therefore, quiescence tends to be more common in later phases of an avascular multicellular tumor spheroid. Quiescence typically increases, often nonlinearly, with tumor size. It can be reversible or irreversible, as reviewed by Skipper (1971).

In an effort to better understand the success of Gompertz models in fitting clinical tumor data, Gyllenberg and Webb (1989) proposed a two-compartment model of the tumor cells in a typical avascular multicellular tumor spheroid transition into and out of quiescence. We briefly describe their model below. Let $P(t)$ and $Q(t)$ be the densities of proliferative and quiescent cells, respectively. Define $N(t)=P(t)+Q(t)$. Then the Gyllenberg-Webb model (GW model) takes the following form:

$$
\left\{\begin{array}{l}
\frac{\mathrm{d} P}{\mathrm{~d} t}=\left(\beta-\mu_{p}-r_{0}(N)\right) P+r_{i}(N) Q \\
\frac{\mathrm{d} Q}{\mathrm{~d} t}=r_{0}(N) P-\left(r_{i}(N)+\mu_{q}\right) Q
\end{array}\right.
$$

with initial conditions,

$$
P(0)=P_{0}>0, \quad Q(0)=Q_{0} \geq 0 .
$$

Proliferative cells are assumed to proliferate at a constant per capita rate $\beta>0$, and proliferative and quiescent cells are assumed to die at constant rates $\mu_{p} \geq 0$ and $\mu_{q} \geq 0$, respectively. The key assumption of Gyllenberg-Webb model is the tumor cells transition to and from the quiescent compartment at rates $r_{0}(N)$ and $r_{i}(N)$, respectively, where both functions are continuous and defined for $N \geq 0$. Recall that stress often increases with tumor size and stressed cells tend to enter and stay at a quiescent state. Gyllenberg and Webb assume that

(A1): $r_{0}(N) \geq 0$ and $r_{0}^{\prime}(N) \geq 0$ for all $N>0$ and,

$$
0<\lim _{N \rightarrow \infty} r_{0}(N) \equiv l_{0} \leq \infty
$$

(A2): $r_{i}(N) \geq 0$, but $r_{i}^{\prime}(N) \leq 0$, and

$$
0 \leq \lim _{N \rightarrow \infty} r_{i}(N) \equiv l_{i}<\infty
$$

(A3): The dead cells are immediately removed from the tumor.

It can be shown that under reasonable conditions, solutions of the above Gyllenberg and Webb model, like that of Gompertz model, take the form of an S-shaped growth curve. 
In this paper, we would like to make two natural modifications to the Gyllenberg-Webb model (2.1). Specifically, we would like to include the dead cell population $D(t)$ into the model. We assume that dead cells are removed from the tumor at a constant rate $d$. For convenience, we will denote $\beta-\mu_{p}$ by $b$ and $\mu_{q}$ by $\mu$. All parameters below are nonnegative constants. This may result in the following three-compartment model.

$$
\left\{\begin{aligned}
\frac{\mathrm{d} P}{\mathrm{~d} t} & =\left(b-r_{0}(N)\right) P+r_{i}(N) Q \\
\frac{\mathrm{d} Q}{\mathrm{~d} t} & =r_{0}(N) P-\left(r_{i}(N)+\mu\right) Q \\
\frac{\mathrm{d} D}{\mathrm{~d} t} & =\mu Q-d D
\end{aligned}\right.
$$

where

$$
N(t)=P(t)+Q(t)+D(t)
$$

and initial conditions are,

$$
P(0)=P_{0}>0, \quad Q(0)=Q_{0} \geq 0, \quad D(0)=Q_{0} \geq 0 .
$$

Here are some examples of functions $r_{0}(N)$ and $r_{i}(N)$

$$
r_{0}(N)=k N, k \ln (1+N), \frac{k N}{a N+1}, \frac{k N^{2}}{a N^{2}+1}
$$

and

$$
r_{i}(N)=\frac{r}{N+m}, \frac{r}{N^{2}+m} .
$$

Throughout the rest of this paper, we assume that the assumptions (A1) and (A2) hold and the parameters $b, \mu, d$ are positive.

\section{Fundamental model properties}

In this section, we present a preliminary analysis of the basic properties such as positivity and boundedness of solutions for the three-compartment model (2.5). We also briefly consider the existence and locations of nonnegative steady states of the model which enables us to appreciate how the steady state tumor size and structure are affected by model parameters and functions and hence allows us to gain some novel biological insights of avascular tumor growth. 
3.1. Positiveness of solutions. It is easy to see from the model equations, if the tumor initially has only proliferative cells, then in an infinitesimal time, some proliferative cells will enter the quiescent state, and some proliferative and quiescent cells will die in the same period. This indicates that without loss of generality, we can assume initial values for $P, Q$ and $D$ populations are positive. The following theorem confirms that with positive initial values, the solutions of model (2.5) are confined to a biologically meaningful region.

Proposition 3.1. Solutions of model (2.5) with positive initial values will stay positive.

Proof. If the proposition is false, then at some time the solution turns negative, then there is a first time $t_{1}>0$ that one of the three solution components becomes zero. Without loss of generality, assume that $P\left(t_{1}\right)=0$ and $\min \{P(t), Q(t), D(t)\}>0$ for $t \in\left(0, t_{1}\right)$. Let $k_{p} \equiv \min \left\{b-r_{0}(N), t \in\left[0, t_{1}\right]\right\}$. Then,

$$
\frac{\mathrm{d} P}{\mathrm{~d} t} \geq k_{p} P, \quad t \in\left[0, t_{1}\right]
$$

which implies that

$$
P(t) \geq P(0) e^{k_{p} t}>0, \quad t \in\left[0, t_{1}\right],
$$

a contradiction to the assumption that $P\left(t_{1}\right)=0$. Similar contradictions can be derived if $Q\left(t_{1}\right)=0$ or $D\left(t_{1}\right)=0$ for some time $t_{1}>0$. This completes the proof of the proposition.

3.2. Boundedness of solutions. Intuitively, the Gyllenberg-Webb model (2.1) can be viewed as the limiting case of the model (2.5) as $D$ tends to infinity. It is easy to see that if $l_{0}<b$, then $\frac{\mathrm{d} P}{\mathrm{~d} t} \geq\left(b-l_{0}\right) P$ which implies that $P(t) \geq P(0) e^{\left(b-l_{0}\right) t}$. Hence, some condition must be placed on $l_{0}$ to ensure that the tumor growth eventually comes to an end. The question is what other conditions shall we add to establish the boundedness of the solutions of the model (2.5). A relatively straightforward set of conditions will be $r_{i}(N) \equiv 0$ and $l_{0}>b$, which is probably reasonable biologically since as tumor grows, there are negligible amount of quiescent cells can reenter proliferative state. This set of conditions are used to establish boundedness of solutions for the model (2.1) in the Proposition 2.1 of Gyllenberg and Webb (1989). In the following proposition, we show that the strict requirement of $r_{i}(N) \equiv 0$ can be replaced by an equally simple yet more general and realistic condition that $r_{i}(N) N$ is bounded. With this assumption, we show below that the solutions of model (2.5) are eventually uniformly bounded. 
Theorem 3.1. Assume that $b<l_{0}<\infty$ and there is a constant $I>0$ such that $r_{i}(N) N<I$ for all $N>0$. Then, solutions of model (2.5) are bounded. Moreover, there is a constant $L>0$ such that

$$
\limsup _{t \rightarrow \infty} N(t) \leq L
$$

Proof. We first show that $P$ must be bounded. Since $l_{0}>b$, for any $\varepsilon_{1}>0$, there is a positive constant $K_{1}$ such that $N \geq K_{1}$ implies that $r_{0}(N)>b+\varepsilon$. Since $Q \leq N$, it is easy to see that

$$
\frac{\mathrm{d} P}{\mathrm{~d} t}<\left(b-r_{0}(N)\right) P+I .
$$

We claim that

$$
P<\max \left\{K_{1}, P(0), I / \varepsilon_{1}\right\}+1 \equiv K_{P} .
$$

If not, then there is a time $t_{1}>0$ such that $P\left(t_{1}\right)=K_{P}, P^{\prime}\left(t_{1}\right) \geq 0$ and $P(t)<K_{P}$ for $0 \leq t<t_{1}$. However, since $N \geq P$, we have

$$
\frac{\mathrm{d} P}{\mathrm{~d} t}\left(t_{1}\right)<\left(b-r_{0}\left(N\left(t_{1}\right)\right)\right) P\left(t_{1}\right)+I<-\varepsilon_{1} P\left(t_{1}\right)+I<0,
$$

a contradiction. Our proof above also implies that

$$
\limsup _{t \rightarrow \infty} P(t) \leq \max \left\{K_{1}, I / \varepsilon_{1}\right\} \equiv L_{P} .
$$

Next, we show that $Q$ is also bounded. In fact, for any $\varepsilon_{2}>0$, we can show that

$$
Q<\max \left\{l_{0} K_{P} / \mu, Q(0)\right\}+\varepsilon_{2} \equiv K_{Q} .
$$

If not, then there is a time $t_{2}>0$ such hat $Q\left(t_{2}\right)=K_{Q}, Q^{\prime}\left(t_{2}\right) \geq 0$ and $Q(t)<K_{Q}$ for $0 \leq t<t_{2}$. However, since $r_{0}(N)<l_{0}$, we have

$$
\frac{\mathrm{d} Q}{\mathrm{~d} t}\left(t_{2}\right)<r_{0}(N) P-\mu Q<l_{0} K_{P}-\mu Q\left(t_{2}\right)<0,
$$

also contradiction. Standard comparison argument can show that

$$
\limsup _{t \rightarrow \infty} Q(t) \leq l_{0} L_{P} / \mu \equiv L_{Q}
$$

Similarly, we can show that $Q$ is bounded and $\lim _{\sup _{t \rightarrow \infty}} D(t) \leq \mu L_{Q} / d \equiv$ $L_{D}$. It is now straightforward to see that

$$
\limsup _{t \rightarrow \infty} N(t) \leq L_{P}+L_{Q}+L_{D} \equiv L
$$

This completes the proof of the theorem.

The following corollary is the result of a simple application of the above boundedness theorem. 
Corollary 3.1. Assume that $r_{0}(N)=\frac{k N}{a N+1}, r_{i}(N)=\frac{r}{N+m}$, and $b<k / a$, where all parameters are positive. Then, solutions of model (2.5) are bounded. Moreover,

$$
\limsup _{t \rightarrow \infty} N(t) \leq \frac{b}{k-b a}\left(1+\frac{k}{a}\left(\frac{1}{\mu}+\frac{1}{d}\right)\right) .
$$

Clearly, Theorem 3.1 does not cover the case of $r_{0}(N)=k N$. The difficulty stems from the fact that the recruitment to the quiescent population is pushed away from $P$, not generated by $Q$. This case is deceivingly simple. It requires a different and more elaborate treatment.

Proposition 3.2. Assume that $r_{0}(N)=k N, k>0$, and there is a constant $I>0$ such that $r_{i}(N) N(1+N)<I$ for all $N>0$. Then, solutions of model (2.5) are bounded.

Proof. Since there is a constant $I>0$ such that $r_{i}(N) N(N+1)<I$ for all $N>0$, we have $r_{i}(N) N<I$ and $r_{i}(N) N^{2}<I$ for all $N>0$. In fact, we claim that

$$
P \leq \max \left\{\frac{1+b}{k}, P(0), I\right\} \equiv P_{m}
$$

If Eq. (3.1) is false, then there is a time $t_{1}>0$ such that $P\left(t_{1}\right)=P_{m}, P^{\prime}\left(t_{1}\right) \geq$ 0 and $P(t)<P_{m}$ for $0 \leq t<t_{1}$. However, since $N \geq P$, we have

$$
\frac{\mathrm{d} P}{\mathrm{~d} t}\left(t_{1}\right)<\left(b-r_{0}\left(N\left(t_{1}\right)\right)\right) P\left(t_{1}\right)+I \leq-P\left(t_{1}\right)+I \leq 0,
$$

which is a contradiction. This proves that the Eq. (3.1) is true.

Next, we show that the expression $Z(t) \equiv N(t) P(t)$ is bounded. Observe that $N Q<N^{2}$. We have

$$
Z^{\prime}=(b P-d D) P+(b-k N) Z+r_{i}(N) N Q<I+b P^{2}-(k N-b) Z .
$$

We claim that

$$
Z(t) \leq \max \left\{I+(b+1)\left(\frac{1+b}{k}\right)^{2}, P^{2}(0)\right\} \equiv U .
$$

If Eq. (3.3) is false, then there is a time $t_{2}>0$ such that $Z\left(t_{2}\right)=U, Z^{\prime}\left(t_{2}\right) \geq 0$ and $Z(t)<U$ for $0 \leq t<t_{2}$. Since $P(t) \leq \frac{1+b}{k}$, we must have $N\left(t_{2}\right)>\frac{1+b}{k}$. We thus have

$$
Z^{\prime}\left(t_{2}\right)<I+b P^{2}-\left(k N\left(t_{2}\right)-b\right) Z\left(t_{2}\right)<I+b\left(\frac{1+b}{k}\right)^{2}-U<0
$$

which is also a contradiction. This proves that the Eq. (3.3) is true. 
Since $Q^{\prime} \leq k Z-\mu Q$ and $Q(0)=0$, a standard comparison argument yields that

$$
Q(t) \leq k U / \mu \text {. }
$$

Likewise, we have $D \leq k U / d$. This completes the proof of the proposition.

3.3. Existence and locations of nonnegative steady states. We now consider the existence and locations of nonnegative steady states of model $(2.5)$. Clearly $(0,0,0)$ is the trivial steady state. Let $E^{*}=\left(P^{*}, Q^{*}, D^{*}\right)$ be a nontrivial steady state of model (2.5).

It is easy to see that

$$
\frac{\mathrm{d} N}{\mathrm{~d} t}=\underline{\mathrm{P}}-d D \text {. }
$$

At steady state, we must have $b P^{*}-d D^{*}=0$. From the $D$ equation, we have $\mu Q^{*}-d D^{*}=0$. Hence $b P^{*}=d D^{*}=\mu Q^{*}$ and

$$
N^{*}=P^{*}+Q^{*}+D^{*}=\left(1+\frac{b}{\mu}+\frac{b}{d}\right) P^{*} \equiv \delta P^{*} .
$$

From the $Q$ equation, we have

$$
r_{0}\left(N^{*}\right) P^{*}=\left(r_{i}\left(N^{*}\right)+\mu\right) Q^{*}=\left(r_{i}\left(N^{*}\right)+\mu\right) \frac{b}{\mu} P^{*},
$$

which implies that

$$
\mu r_{0}\left(N^{*}\right)=b\left(r_{i}\left(N^{*}\right)+\mu\right) .
$$

We are now in a position to state and prove the following result on the existence and uniqueness of positive steady state for model (2.5).

Proposition 3.3. Assume the following two conditions hold

$$
\mu r_{0}(0)<b\left(r_{i}(0)+\mu\right),
$$

and there is a positive constant $U$ such that

$$
\mu r_{0}(U)>b\left(r_{i}(U)+\mu\right) .
$$

Then, model (2.5) has a unique positive steady state.

Proof. The proof of this proposition is straightforward. Let

$$
f(N) \equiv \mu r_{0}(N)-b\left(r_{i}(N)+\mu\right) .
$$

We see that $f(N)$ is strictly increasing with $f(0)<0$ and $f(U)=\mu r_{0}(U)-$ $b\left(r_{i}(U)+\mu\right)>0$. Hence, there is a unique $N^{*} \in(0, U)$ such that $f\left(N^{*}\right)=0$. The unique positive steady state is given by

$$
E^{*}=\left(\frac{N^{*}}{\delta}, \frac{b N^{*}}{\mu \delta}, \frac{b N^{*}}{d \delta}\right) .
$$


This completes the proof of the proposition.

For the case of $r_{0}(N)=\frac{k N}{a N+1}$ and $r_{i}(N)=\frac{r}{N+m}$, where all parameters are positive, the above proposition ensures the solutions of model (2.5) possess a unique positive steady state if there is a $U>0$ such that

$$
\mu \frac{k U}{a U+1}>b\left(\frac{r}{U+m}+\mu\right) .
$$

In case of $a=m=1$, this reduces to the existence of $U>0$ such that $\mu[(k-b) U-b]>b r$, which is easily satisfied if $k>b$. From Proposition 3.1, we also see that the solutions of model (2.5) are also bounded.

3.4. Steady state tumor size and structure. It is worthy noting that at steady state, the tumor size $N^{*}$ does not depend on the dead cell removal rate $d$, while the ratio of proliferative cells to the total tumor cells is $1 / \delta$ which does not depend on the transfer rate functions $r_{0}(N)$ and $r_{i}(N)$. Moreover, as $\mu$ tends to zero, we see that $\delta$ tends to infinity from (3.4), which implies that at the proliferative portion of the tumor approaches to zero at or near steady state level. This echoes a similar statement included in the Proposition 2.3 of Gyllenberg and Webb (1989). Moreover, it is easy to see that $f\left(N^{*}\right)=0$ implies that

$$
b=\frac{\mu r_{0}\left(N^{*}\right)}{\mu+r_{i}\left(N^{*}\right)}<r_{0}\left(N^{*}\right) .
$$

By implicitly differentiating the equation $\mu r_{0}(N)-b\left(r_{i}(N)+\mu\right)=0$ with respect to $\mu$, we can show that

$$
\frac{\mathrm{d} N^{*}}{\mathrm{~d} \mu}=\frac{b-r_{0}\left(N^{*}\right)}{r_{0}^{\prime}\left(N^{*}\right)-b r_{i}^{\prime}\left(N^{*}\right)}<0 .
$$

These elementary mathematical findings can be summarized into the following biological proposition on the steady state tumor size and structure.

Proposition 3.4. If an avascular tumor grows following the model (2.5), then as the death rate of quiescent cells decreases to zero, the percentage of proliferative cells also approaches to zero. Moreover, a slow dying quiescent population will increase the size of the tumor at the steady state level.

\section{Stability of Steady States}

At the onset of tumor growth, plenty of nutrient is available for tumor cells to proliferate, hence there is no need for cell to enter the quiescent state. In view of this fact, we assume in the rest of this paper that

$(\mathbf{A} 4): r_{0}(0)=0$. 
We will study the stability of the trivial steady state and the nontrivial steady state when it exists.

We consider first the local stability of $E_{0}=(0,0,0)$ which is determined by the following Jacobian,

$$
J\left(E_{0}\right)=\left[\begin{array}{ccc}
b & r_{i}(0) & 0 \\
0 & -r_{i}(0)-\mu & 0 \\
0 & \mu & -d
\end{array}\right] .
$$

It is easy to see that $J\left(E_{0}\right)$ has eigenvalues $b,-r_{i}(0)-\mu$ and $-d$, implying it is a saddle.

We now consider the local stability of $E^{*}=\left(P^{*}, Q^{*}, D^{*}\right)$ which is determined by the following Jacobian,

$$
J \equiv J(E *)=\left[\begin{array}{ccc}
-A-B & -A+C & -A \\
A+B & A-C-\mu & A \\
0 & \mu & -d
\end{array}\right]
$$

where

$$
A=r_{0}^{\prime}\left(N^{*}\right) P^{*}-r_{i}^{\prime}\left(N^{*}\right) Q^{*}>0, B=r_{0}\left(N^{*}\right), C=r_{i}\left(N^{*}\right) .
$$

From Routh-Hurwitz criterion, we know that all eigenvalues of $J$ have negative real parts if all the following three conditions hold:

(i) $\operatorname{tr} J<0, \quad($ ii $) \operatorname{det} J<0, \quad($ iii $) \Delta \equiv \operatorname{det} J-(\operatorname{tr} J)\left(\sum_{k=1}^{3} A_{k k}\right)>0$,

where $A_{k k}$ is the determinant of the $2 \times 2$ matrix obtained by removing the $k$-th row and $k$-th column from $J$ (see page 234 of Edelstein-Keshet, 1988). The following theorem gives a set of sufficient conditions for the local stability of $E^{*}$.

From Eq. (3.10), we see that $b<r_{0}\left(N^{*}\right)=B$. Hence

$$
\operatorname{tr}(J)=b-B-C-\mu-d<0 .
$$

Straightforward computation yields

$$
\operatorname{det}(J)=b d C-d \mu(B-b)-A(b d+b \mu+\mu d),
$$

and

$A_{11}=d(C+\mu-A)-\mu A, \quad A_{22}=d(A+B-b), \quad A_{33}=\mu(A+B)+b(A-C)-\mu b$.

From Eq. (3.6), we see that $\mu B=b(C+\mu)$ which implies that

$$
\operatorname{det}(J)=-A(b d+b \mu+\mu d)<0,
$$

$$
A_{33}=\mu(A+B)+b(A-C)-\mu b=A(\mu+b)>0
$$


and

$$
A_{11}+A_{22}+A_{33}=d(B-b)+d \mu+d C+b A>0
$$

Hence,

$$
\Delta=-A(b d+b \mu+\mu d)+[B-b+C+\mu+d][d(B-b)+d \mu+d C+b A] .
$$

Therefore, we have established the following local stability result for the positive steady state $E^{*}$.

Theorem 4.1. $E^{*}$ of the model (2.5) is locally asymptotically stable if and only if

$$
[B-b+C+\mu+d][d(B-b)+d \mu+d C+b A]>A(b d+b \mu+\mu d) .
$$

Notice that the tumor size at the steady state $N^{*}$ does not depend on $d$, nor the values of $A, B$ and $C$. These facts imply that the condition of Eq. (4.9) holds for large values of $d$. In the limiting case of $d=\infty$, our model reduces to the model (2.1). In other words, the above theorem implies that the positive steady state in the model of Gyllenberg and Webb (1989) is always asymptotically stable when exists.

In real avascular tumor growth, one can probably neglect the amount of cells that reenter the proliferative state from the quiescent state. That is, we can assume that $r_{i}(N) \equiv 0$. In this special case $\equiv B$. The above Theorem can be reduced to

Proposition 4.1. Assume that $r_{i}(N) \equiv 0$ in model (2.5). Then, $E^{*}$ is locally asymptotically stable if and only if

$$
\mu+d>r_{0}^{\prime}\left(N^{*}\right) P^{*}
$$

One can see from the model (2.5) that the value $N^{*}$ is independent of the parameters $\mu$ and $d$. Moreover, the value of $P^{*}$ is given by Eq. (3.4) which indicates that it does not depend on the value of $r_{0}^{\prime}\left(N^{*}\right)$. In other words, mathematically, one can formulate function $r_{0}(N)$ so that $\mu+d<r_{0}^{\prime}\left(N^{*}\right) P^{*}$ and hence generate an unstable positive steady state. However, when $r_{i}(N) \equiv$ 0 and $r_{0}(N)=\frac{k N}{a N+1}$, then the positive steady state of the model $(2.5)$ always exists and is locally asymptotically stable. Observe that when $a=0$, we have $r_{0}(N)=k N$.

Proposition 4.2. Assume that $r_{i}(N) \equiv 0$ in model (2.5). Then, $E^{*}$ exists and is locally asymptotically stable if $r_{0}(N)=\frac{k N}{a N+1}$. 

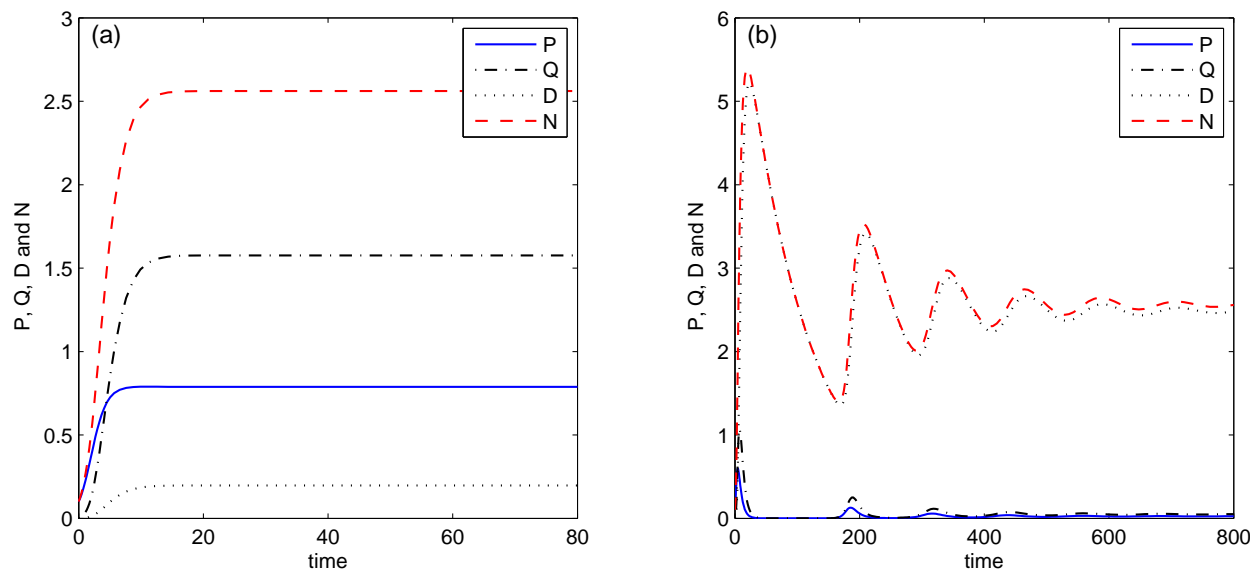

Figure 2. Two sets of solutions of model (2.5) with $r_{i}(N)=\frac{r}{N+m}$, and $r_{0}(N)=\frac{k N}{a N+1}$. Except for the dead cell removal rate $d$, the parameters for both panels are the same. They are $b=1, k=2, a=1, m=2, \mu=$ $0.5, r=1$. For panel (a), $d=4$ and for panel (b), $d=0.01$. The initial conditions are $P(0)=0.1, Q(0)=D(0)=0$ for both panels.

Proof. Assume that $r_{0}(N)=\frac{k N}{a N+1}$. Then, $N^{*}=\frac{b}{k-a b}, P^{*}=\frac{N^{*}}{\delta}$, and Eq. (4.10) is equivalent to

$$
\mu+d>\frac{b(k-a b)}{\delta k}
$$

where $\delta=1+\frac{b}{\mu}+\frac{b}{d}$. The above inequality is equivalent to

$$
(\mu+d)\left(1+\frac{b}{\mu}+\frac{b}{d}\right)>\frac{(k-a b)}{k},
$$

which is obviously true. This completes the proof.

Indeed, our extensive simulation and bifurcation analysis suggest that $E^{*}$ is globally asymptotically stable when $r_{i}(N)=\frac{r}{N+m}, r_{0}(N)=\frac{k N}{a N+1}$ and $k>a b$ in model (2.5) (see Figures 2 and 3). However, the model (2.5) does not possess any monotonicity or generate monotone solution components to allow standard global stability analysis that involves monotone system theory or Lyapunov functions. We leave this as an open question. 


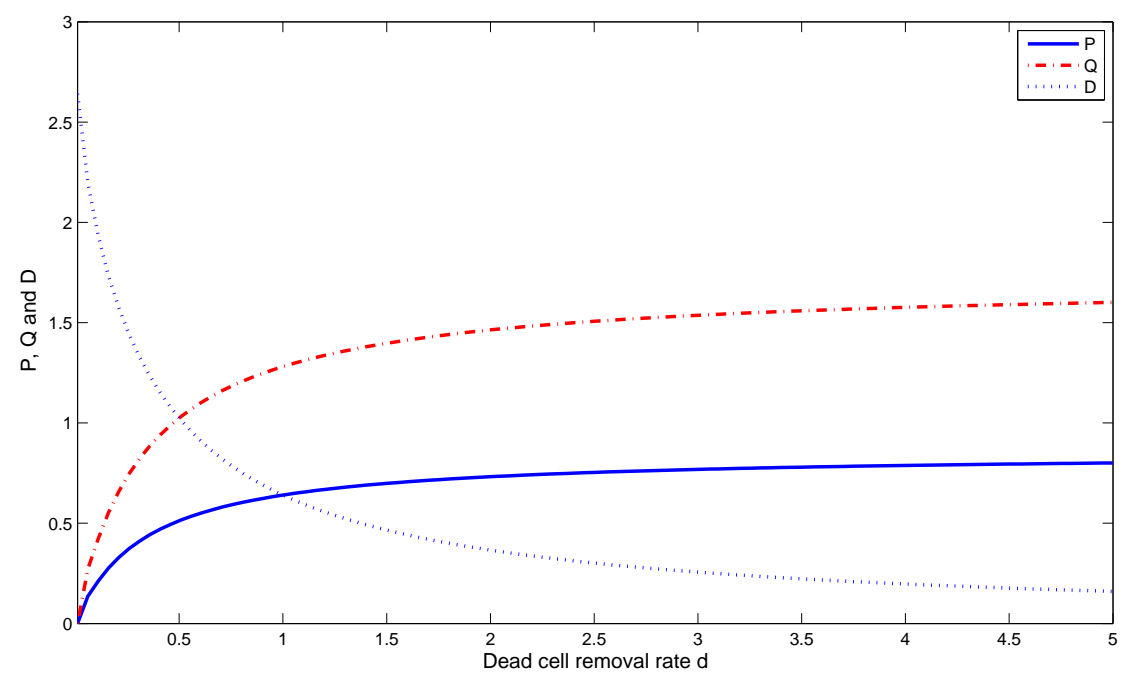

Figure 3. Bifurcation diagrams of model (2.5) with $r_{i}(N)=\frac{r}{N+m}$, and $r_{0}(N)=\frac{k N}{a N+1}$ using the dead cell removal rate $d$ as the bifurcation parameter. The parameter values are $b=1, k=2, a=1, m=2, \mu=0.5, r=$ 1. Clearly, the positive steady state appears to be globally attractive and the components of the steady state are monotone with respect to $d$.

\section{Discussion}

As pointed out in the introduction, the Gyllenberg-Webb model (2.1) is essentially the limit case of the model (2.5) by taking $d=\infty$. Biologically $d=\infty$ is equivalent to assuming the dead cells are removed from the tumor instantly which is far from the truth and ignores the existence of a necrotic core in most avascular tumors in their late stage. By including the dead cell population remaining in the tumor explicitly, we arrive at the three-population model (2.5) which is deceivingly simple-looking mathematically. While it does allow interesting and fundamental mathematical analysis of the basic model properties, it defies our initial efforts in establishing nontrivial global stability results for the positive steady state even for the simple case of $r_{i}(N)=0$, and $r_{0}(N)=k N$.

A nonintuitive observation resulted from the model (2.5) is that the subpopulation ratios in an avascular tumor at the steady state level do not depend on the cell state transition functions $r_{0}(N)$ and $r_{i}(N)$. In other words, avascular tumors of average proliferation rate, quiescent cell death rate and dead cell removal rate shall have the same subpopulation ratios. 
A probably more intuitive but interesting observation resulted from our basic model properties is that tumor final size, if represented by the positive steady state, does not depend on the dead cell removal rates. The medical implication of this observation is that for a solid tumor that does not allow easy removal of dead cells naturally or by the help of macrophages, the tumor will have a larger necrotic core, while a more loosely assembled tumor may have a small or no necrotic core.

The case of $r_{i}(N)=\frac{r}{N+m}$ and $r_{0}(N)=\frac{k N}{a N+1}$ for model (2.5) is probably most intriguing mathematically and representative biologically. In Figure 2 , we present two sets of solutions of model (2.5) with different dead cell removal rates. Observe that for larger dead cell removal rates, all the components as well as the tumor size grow like a sigmoid curve, which is the hallmark of Gompertz tumor growth. However, for small $d$ values, the solution components are oscillatory for a period of time. This transitional dynamics suggests that oscillatory tumor sizes maybe caused by low dead cell removal rate that maybe the result of tight tumor capsule or inhibited macrophage activities.

As for any cells, tumor cells are limited by resources such as nutrient, growth signals, or space, or all of these. However, for most tumors, the most limiting element is most likely a key nutrient. It is thus important for tumor models to implicitly or explicitly include such limiting resources. This is attempted in some recent tumor modeling efforts (Gyllenberg and Webb (1990), Eikenberry et al. (2009), Eikenberry et al. (2009a), Eikenberry et al. (2010), Thalhauser et al. (2009)), some of which are also preliminarily validated by clinical data (Portz et al. (2012); Everett et al. (2013)). For incrementally more realistic models, one can consider incorporating time delays in the cell transition and cell death processes. If space is limiting or spatial distribution must be modeled, then one shall consider employing partial differential equation models. All these considerations call for more observation, data or hypothesis based modeling efforts.

\section{ACKNOWLEDGMENTS}

This work was funded by the Deanship of Scientific Research (DSR), King Abdulaziz University, under grant No. (16-130/1433 HiCi). The authors, therefore, acknowledge the technical and financial support of KAU.

\section{REFERENCES}

[1] Bertalanffy L von: Quantitative laws in metabolism and growth. Quart. Rev. Biol., 1957, 32:217-231.

[2] Edelstein-Keshet L: Mathematical models in biology. 1988. SIAM. 
[3] Eikenberry SE, Sanker T, Preul MC, Kostelich EJ, Thalhauser C, Kuang Y: The virtual glioblastoma: growth, migration, and treatment in a three-dimensional mathematical model, Cell Proliferation, 2009, 42:511-528. (pdf 987k). DOI: 10.1111/j.13652184.2009.00613.x

[4] Eikenberry SE, Thalhauser C, Kuang Y: Tumor-Immune Interaction, Surgical Treatment, and Cancer Recurrence in a Mathematical Model of Melanoma, PLoS Comput. Biol., 2009a, 5(4): e1000362. doi:10.1371/journal.pcbi.1000362.

[5] Eikenberry SE, Nagy JD, Kuang Y: The evolutionary impact of androgen levels on prostate cancer in a multi-scale mathematical model, Biology Direct, 2010, 5:24 doi:10.1186/1745-6150-5-24.

[6] Everett RA, Zhao Y, Flores KB, Kuang Y: Data and implication based comparison of two chronic myeloid leukemia models, Math. Biosc. Eng., 2013, 10:1501-1518.

[7] Frenzen CL, Murray JD: A cell kinetics justification for Gompertz equation, SIAM J. Appl. Math., 1986, 46:614-629.

[8] Gompertz B: On the nature of the function expressive of the law of human mortality, and on a new mode of determining the value of life contingencies. Phil. Trans. Royal Soc. London, 1825, 115:513-583.

[9] Gyllenberg M, Webb GF: Quiescence as an explanation of Gompertzian tumor growth. Growth Dev Aging, 1989, 53:25-33.

[10] Gyllenberg M, Webb GF: A nonlinear structured population model of tumor growth with quiescence. J Math. Biol., 1990, 28:671-694.

[11] Kozusko F, Bajzer Z: Combining Gompertzian growth and cell population dynamics, Math. Biosc., 2003, 185:153-167.

[12] Laird AK: Dynamics of tumor growth. Brit. J. Cancer, 1964, 18:490-502.

[13] Marusic M, Vuk-Pavlovic S: Prediction power of mathematical models for tumor growth, Journal of Biological Systems, 1993, 1:69-78.

[14] Marusic M, Bajzer Z, Vuk-Pavlovic S, Freyer JP: Tumor growth in vivo and as multicellular spheroids compared by mathematical models, Bull. Math. Biol., 1994, 56:617-631

[15] Norton L, Simon R, Brereton HD, Bogden AE: Predicting the course of Gompertzian growth. Nature 1976, 264:542-545.

[16] Portz T, Kuang Y, Nagy JD: A clinical data validated mathematical model of prostate cancer growth under intermittent androgen suppression therapy, AIP Advances, 2012, 2:011002; doi: $10.1063 / 1.3697848$

[17] Skipper HE: Kinetics of mammary tumor cell growth and implications for therapy. Cancer, 1971, 28:1479-99.

[18] Thalhauser CJ, Sankar T, Preul MC, Kuang Y: Explicit separation of growth and motility in a new tumor cord model, Bulletin of Math. Biol., 2009, 71:585-601. 